\title{
Development of an artificial neural network for helping to diagnose diseases in urology
}

\author{
Antonio Soriano Payá, Daniel Ruiz Fernández, David Gil Méndez, Carlos Alberto Montejo Hernández \\ Computing Technology and Data Processing, University of Alicante \\ Alicante, Spain \\ \{soriano, druiz, dgil, cmontejo\}@dtic.ua.es
}

\begin{abstract}
In this article we propose the development of a tool for helping in the medical diagnosis using neural networks, in particular the multilayer perceptron. This new tool is meant to help the urologists in obtaining an automatic diagnosis for complex multi-variable systems, and to avoid painful and costly medical treatments. The clinical study has been carried out using the medical registers of patients with dysfunctions in the lower urinary tract. The system is able to distinguish and classify dysfunctions as arreflexive, hyper-reflexive, and effort incontinence. Moreover, it is able to predict whether there is presence of dysfunction or not. The results of the experiments display a high percentage of certainty of about $85 \%$.
\end{abstract}

Keywords-component; Urology, artificial neural network, decision support systems.

\section{INTRODUCTION}

Apart from the different tools available for the urologists to obtain urodynamical data, it still remains very complicated to lead to a correct diagnosis: the knowledge concerning the origin of the detected dysfunctions depends mainly on acquired experience and on the research which is constantly carried out within the field of urology. The experts are often confronted to situations that are poorly described or that are not described in the medical bibliography. Also, the dysfunctions whose precise diagnoses are complicated are numerous as consequence of the interaction with the neural system and the limited knowledge available on how it operates.

For the purpose of diagnosing dysfunctions of the lower urinary tract (LUT) there are various techniques which entail different degrees of invasiveness toward the patient [1]. A urological study of a patient consists of carrying out various costly tests neurological and physical as flowmetry and cystometry examinations with a high degree of complexity and of invasiveness. This project is intended to aid the specialist in obtaining a reliable diagnosis with the smallest possible number of tests. This way, major benefits are obtained both for the patient, avoiding him painful tests, and for the medical centres, avoiding expensive urodynamical tests and reduce waiting lists.

Decision support systems (DSS) in medicine can be viewed as intelligent advisors, or sources of second opinion. Their typical life cycle often consists of defining a problem on which to focus, gathering the corresponding retrospective data, and constructing the predictive model. A decision support system has to be immediately accessible providing the useful data in accordance with the situation [2][3]. The range of techniques used as tools for a DSS is very wide, covering everything from traditional statistics and expert systems (ES) to more emerging fields such as Artificial Neural Networks (ANN), Fuzzy Inference Systems (FIS) [4] and other technologies within Soft Computing [5].

In the present article is implemented a system for aiding in diagnosing dysfunctions of the LUT. The remaining part of the paper is organized as follows: first, it describes the design of a DSS and a brief state of the art of applications based on DSS. Next, it describes the design of our proposal and its training by means of the available data. Then, it is presented the subsequent testing carried out with new data in order to analyse the results. Finally, the relevant conclusions are drawn.

\section{DECISION SUPPORT SYSTEMS IN MEDICINE}

At the beginning, the use of computer science in diagnosing was oriented to statistical analysis. For example, it is calculated the probability of each sign in the diagnosis and it determined the most indicated. Later, the ES appeared, in which there is an interaction of the doctor with the computer. The ES is a program based on the knowledge, which emulates a human expert in the resolution of a significant problem in a specific field [6]. The characteristic of the processes of an ES is to simulate the clinical judgment and to use a mathematical schematization of the medical thought. These systems are used as DSS, both diagnosis and therapeutic, and they are never assumed as substitutes of the doctor. The aim for using these systems is the constant increase of the amount of knowledge and data to consider in a decision, due to the great technological advance and to the intense clinical investigation. The capacity of the computers to manage a great amount of knowledge is one of the keys of the success of the ES.

In the last years the increasing development of the DSS has jointed investigators converging from disciplines of artificial intelligence, computer science, mathematical, acquisition of the knowledge, etc.

Some applications that have been developed for the DSS are: 
- $\quad$ ERA. Decision support systems interactive to identify patients with cancer suspicions. Developed in United Kingdom (2001).

- ATHENA. Control of the hypertension in primary cares following medical recommendation of care and medication. Developed in Canada and EEUU (2002).

- LISA. Decision support system for children with lymphoblastique leukaemia. Developed in United Kingdom (2004).

\section{NETWORK SETTING}

The figure shows the different parts of the system.

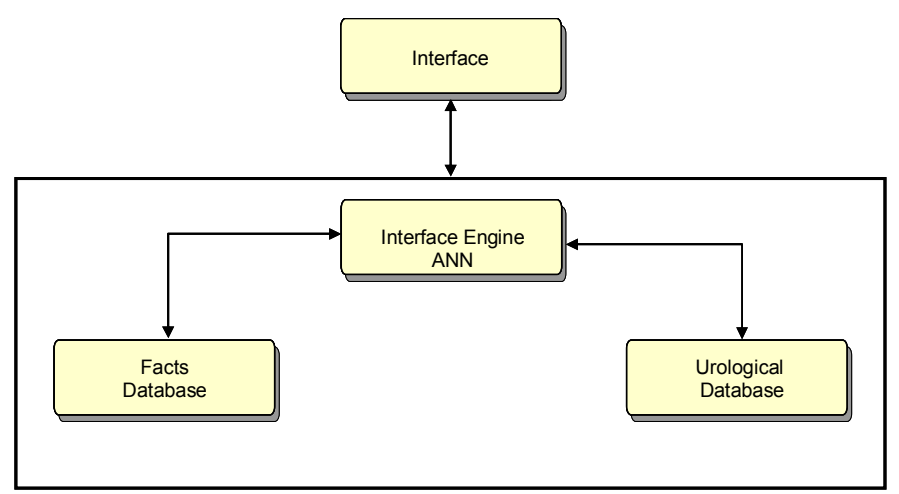

Figure 1. Decision support system (DSS) proposed.

\section{A. The urological database}

The urologist knowledge base stores all the urodynamical measures of about 500 patients stored in a database provided by specialists. As usual operations of maintenance, it is possible to add new registers, to modify or to eliminate.

\section{B. The Facts database}

It is the memory space in which the data received initially are stored. It contains the knowledge on the register in which is working on. Also, we will be registered the intermediate conclusions and the data generated in the inference process. All the intermediate results that are memorized can be used to explain the deductions and the behaviour of the system.

\section{Inference Engine}

In the multidiscipline field of the computer science applied to medicine the central axis of the investigations are in applications of ANN in classification and diagnosis [7] [8]. It is possible to find models with diagnosis benefits, artificial treatment of neuronal dysfunctions and DSS.

The inference engine in our proposal is constructed by using ANN to carry out the reasoning or learning, in particular a multilayer perceptron (MLP) based on the backpropagation algorithm.

The architecture of the ANN (MLP) is the following:
- Layer 1:24 inputs that correspond with data of the data base like result of: (e1-e8) neurological physical exploration, (e9-e13) free flowmetry, (e14-e16) cystometry. (e17-e24) Test pressure detrusor/flow miccional.

- Layer 2: it contains 10 neurons in the hidden layer.

- Layer 3: 3 outputs like result from 3 diagnoses: (d1) Arreflexive, (d2) Hyper-reflexive, (d3) Effort incontinence.

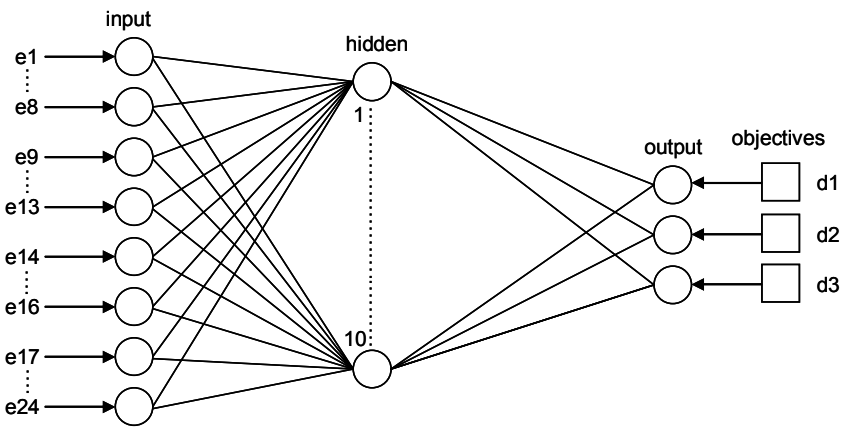

Figure 2. Architecture of the Artificial Neural Network, MLP (input layer, hidden layer and output layer), used for the diagnosis of dysfunctions of the Lower Urinary Tract.

The learning of backpropagation is as it follows:

a) To initialize the weights and the initial thresholds of each neuron: there are several possibilities of initializing being the most common to introduce small random values.

b) For each pattern of the set of the training data:

- To obtain the answer of the network with that pattern. This part is obtained forward propagating the input, since this type of network is feed forward. The outputs of a layer are the neurons of input for the following layer, processing them according to the rule of propagation and the corresponding function of activation.

- To calculate the errors associated by means of the average quadratic error.

- To calculate the partial increases. These increases depend on the errors calculated in the previous step.

c) To calculate the total increase, for all the patterns, of the weights and the thresholds.

d) To update weights and thresholds.

e) To calculate the current error and to return to step 2 if it is not successful.

\section{Interface}

This part is necessary to provide the information to the DSS. The system can be tailored to meet the individual needs of every person. Nowadays, these interfaces are graphical to improve the communication between the user and the system. 


\section{EXPERIMENTATION}

We began with a discretization of our database. There were many problems in the process of discretization because of the differences of ranks, values and types to looking for a way of homogeneity as a previous step in the process of training of the ANN. It was adjusted and weighted with the help of the urologists, following their instructions and suggestions.

For example, some of the fields of the database are sex, age, volume of urine and micturition time. As the differences among all these fields are huge, we created ranks in the values of the fields dividing them in subclasses in order to adjust the data input. For instance:

- $\quad$ Sex: Male (1), Female (2)

- $\quad$ Age: 0-20 (1), 20-50 (2), 50-65 (3), >65 (4)

- Volume of urine: 0-150 (1), 150-300 (2), 300-500 (3), $>500$ (4)

- $\quad$ Micturition time: 0-25 (1), 25-50 (2), 50-75 (3), >75 (4)

Between parenthesis is represented the values discretized (subclasses). As we can observe the difficulties are not only in the data ranks, but also in the types of data which complicates the process of data discretization.

Next step is to run the experimentation. This has been performed by using cross-validation method. Cross validation is a model evaluation method that is better than residuals. The problem with residual evaluations is that they do not give an indication of how well the learner will do when it is asked to make new predictions for data it has not already seen.

The data has been divided in two set. The set of data used for the process of constructing the model (the training data) was of around 400 registers. The other set of data used to validate the model (the test data) was of 100 registers. Since test data are chosen randomly from the initial data and the remaining data form the training data, the name of the method used in this process is the holdout cross-validation. The function approximation fits a function using the training set only. Then the function approximation is asked to predict the output values for the data in the testing set (it has never seen these output values before). The errors it makes are accumulated as before to give the mean absolute test set error, which is used to evaluate the model.

Figure 3 shows the result with the data or validation set (the data used in the testing set). From the graphics, it can be observed that effort incontinence and the disease free patients are the diagnosis with the highest degree of certainty with more than $90 \%$. This is due to the circumstance that the precision, with which the tests carried out were made, specifically within the field of cystometry, results in that the correctly measured value of these fields determines a diagnosis with a very low probability of error. The diagnoses of arreflexive and hyperreflexive represent values of around $80 \%$ accuracy.

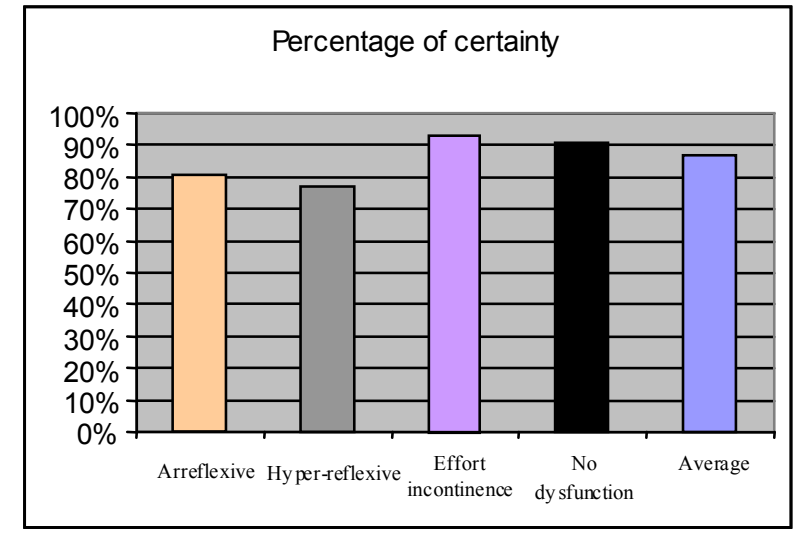

Figure 3. Percentage of certainty of the diagnosis classified according to disease.

\section{CONCLUSIONS}

In the present article a DSS has been developed for the diagnosis of dysfunctions of the lower urinary tract with a degree of certainty of $85 \%$. The system consists of an artificial neural network, multilayer perceptron with the algorithm of learning of backpropagation, as motor of inference. This training for the ANN is made with a database with registers of different patients.

The model starts with a stage of discretization of the urodynamical measures from a patient in order to provide them to the ANN and to determinate if there are any of the three dysfunctions of the LUT or not. In case of finding a dysfunction it would determine what dysfunction or dysfunctions could have.

The research constitutes the basis for further studies in the field of systems for aiding in the diagnosis of dysfunctions of the LUT. In this regard we are considering the use of another type of supervised or unsupervised neural network especially in medical applications [9]. Knowledge-Discovery in Databases (KDD), also known as data mining [10] [11], will be also very demanded when the number of data is huge in order to process automatically searching large volumes of data for patterns.

Moreover, in the process of discretization it will be advantageous to use fuzzy logic because of the differences between the closer subclasses. This process will permit to know more in depth the edge values.

The system is of great help to the urologists as it permits them to eliminate urodynamical tests with different variables and thus saving costs, time and pain for the patients.

\section{ACKNOWLEDGMENT}

The data used in the development of this system is the result of several years of collaboration with urologists of the Hospital of San Juan (Alicante-Spain). The work has been supported by the Office of Science and Technology of the Autonomous Government of Valencia (Spain) as part of the research project "Cooperative diagnosis systems for Urological dysfunctions (2005-2006)". 


\section{REFERENCES}

[1] P. Abrams, "Urodynamics", in Springer, 3rd ed., pp. 20-39, 2005.

[2] L. Bonnevie et al (2005): 'The use of computerized decision support systems in preventive cardiology-principal results from the national PRECARD(R) survey in Denmark', Euro-pean Journal of Cardiovascular Prevention \& Rehabilitation., 12(1), pp. 52-55

[3] E. B. Sloane (2004): 'Using a Decision Support System Tool for Healthcare Technology Assessments', IEEE Engineering in Medicine and Biology Magazine., Vol 23, Numb 3, pp. $42-55$

[4] D. Dumitrescu et al (2000): 'Urodynamics Fuzzy Sets and their Application to Clustering and Training', (Ed): 'CRC Press'

[5] H. Teodorescu, et al (1999): 'Soft Computing in Human-Related Sciences', H. (Ed): 'CRC Press'.
[6] J. Liebowitz. The Handbook of Applied Expert Systems. 1997. (Ed): 'CRC Press', pp. 537.

[7] A. Soriano (2001): 'Modeling and simulation of the neural regulator of the lower urinary tract', Ph.D. Dissertation.

[8] D. Ruiz (2003): "Unsupervised regulation models of biological systems. Description and correction of neurological urinary dysfunctions of human beings", Ph.D. Dissertation.

[9] R. Dybowski, V. Gant, "Clinical Applications of Artificial Neural Networks", Cambridge University Press, 2001.

[10] P. Perner, "Advances In Data Mining: Applications in Image Mining, Medicine and Biotechnology, Management...", Vol. LNAI 3275, Springer , 2004

[11] I. H. Witten, E. Frank, "Data mining", Elsevier , 2005. 